\title{
Active Dosimetry with the Ability to Distinguish Pulsed and Non-Pulsed Dose Rate Contributions
}

\author{
Krystsina Makarevich ${ }^{1}$, Roland Beyer ${ }^{2}$, Jürgen Henniger ${ }^{1}$, Yuzhen $\mathrm{Ma}^{1}$, Sarah Polter ${ }^{1}$, \\ Marian Sommer ${ }^{1}$, Tobias Teichmann ${ }^{1}$, David Weinberger ${ }^{2}$, and Thomas Kormoll ${ }^{1}$ \\ ${ }^{1}$ Technische Univetsität Dresden, Germany \\ ${ }^{2}$ Helmholz Zentrum Dresden-Rossendorf, Germany \\ krystsina.makarevich@tu-dresden.de
}

\begin{abstract}
This paper presents the concept of an active dosimetry system and its operational regime for pulsed radiation dose rate measurements. The plastic scintillator is suggested to be used for absorbed dose rate measurements. As long as the detector can be considered tissue equivalent, the energy weighting of pile-up events in terms of the dose is achieved. The real-time distinction of pulsed and non-pulsed dose rate contributions is based on the time structure of a single interaction and requires only basic information about the beam time structure (pulses duration and period). The detector connected to a fully digital signal processing board creates an active dosimetry system with adjustable parameters. Such a system was used for absorbed dose rate measurements in pulsed photon field mimicking radiation field outside the bunker of a medical LINAC, but also in the presence of a constant radiation component. The results show a linear dependence of a pulsed radiation contribution on the accelerator current in the investigated range of the total dose rate up to $8 \mu \mathrm{Gy} / \mathrm{h}$.
\end{abstract}

Keywords-Active dosimetry, Plastic scintillator, Pulsed radiation, Radiation monitoring.

\section{INTRODUCTION}

$\mathrm{M}$ ANY practical applications (radiation therapy, research accelerators, laser-induced X-rays, etc.) involve pulsed radiation. In such facilities where radiation is generated or used, appropriate dosimetry monitoring has to be accomplished. For continuous radiation one can exploit welltested area monitoring dosimeters based on ionization chambers, semiconductor detectors or proportional counters. But several studies $[1-4]$ revealed that it is not possible to perform accurate dose rate measurements in pulsed radiation fields using the before mentioned dosimeters.

Let us define some criteria that must be satisfied by the dosimeter used for area monitoring in pulsed radiation field. Foremost, such a system has to provide direct readings of the ambient dose or dose rate equivalent [5] and warn users in case of exceeding the established limits. The dosimeter has to be not affected by the pile-up effect, dead-time behavior, energy dependency, or the named effects should be easily corrected. It is also preferable if the dosimeter is fast enough to provide time-resolved measurements necessary, for example, in radiotherapy.

In this regard, plastic scintillators demonstrate several advantages for using them in pulsed radiation dosimetry: they have almost tissue equivalent response to a dose, the results of measurements are not affected by pressure and humidity fluctuations, response time of many of them is in the range of $\mathrm{ns}$, and they can be easily made of a desired shape and size. If appropriate hardware is used, fast scintillators are, in principle, not limited in detecting of pulsed radiation [6].

This paper will give the main considerations regarding the concept of such a detector and its operational regime. The approach of distinguishing pulsed and non-pulsed dose rate contributions will be discussed. The results of dose rate measurements in scattered pulsed radiation field with a time structure imitating the radiotherapeutical beam will be presented and discussed.

\section{MATERIALS AND METHODS}

\section{A. Detector}

To perform ambient dose equivalent measurements needed for area monitoring, one can exploit a tissue equivalent material for the detector. In this case, simultaneous energy depositions in the detector from multiple photons (pile-up) lead to a proportional value of the absorbed dose as from summing up contributions from separately coming photons in soft tissue. This means that pile-up events in the detector material are energy weighted: higher weighting of pile-up events is achieved by their higher energy values. Thus, tissue equivalency of the detector material allows dealing with the pile-up and consequent energy dependence of the detector.

First of all, let's investigate the response of the plastic scintillator materials to the same dose as in soft tissue. For this, the comparison of mass energy-absorption coefficients of vinyltoluene and polystyrene which are polymer bases of many plastic scintillators and ICRU 4-component soft tissue [5] that is used as the reference material for ambient dose equivalents measurements was performed. The results of the comparison are shown in Fig. 1.

In the energy region from $200 \mathrm{keV}$ up to $4 \mathrm{MeV}$, the ratio of the mass energy-absorption coefficients is almost constant. This means that the absorbed dose in a plastic scintillator can be proportionally related to the absorbed dose in soft tissue. 
Beyond this range, plastic scintillators underestimate the absorbed dose up to 2.5 times depending on energy. If one considers dosimetry behind the treatment room, then this behavior is completely satisfactory as the overwhelming part of the photon energy spectrum is in the energy region higher than $100 \mathrm{keV}$ [7, 8]. Regarding laser-induced X-rays, when the energy of incident photons is in the range of tens $\mathrm{keV}$ [9], such a simple correction does not work anymore, and the deconvolution of the spectrum can be required to restore the ratio of mass energy-absorption coefficients. This task is not so trivial as no full absorption peaks are observed in low- $Z$ organic scintillators.

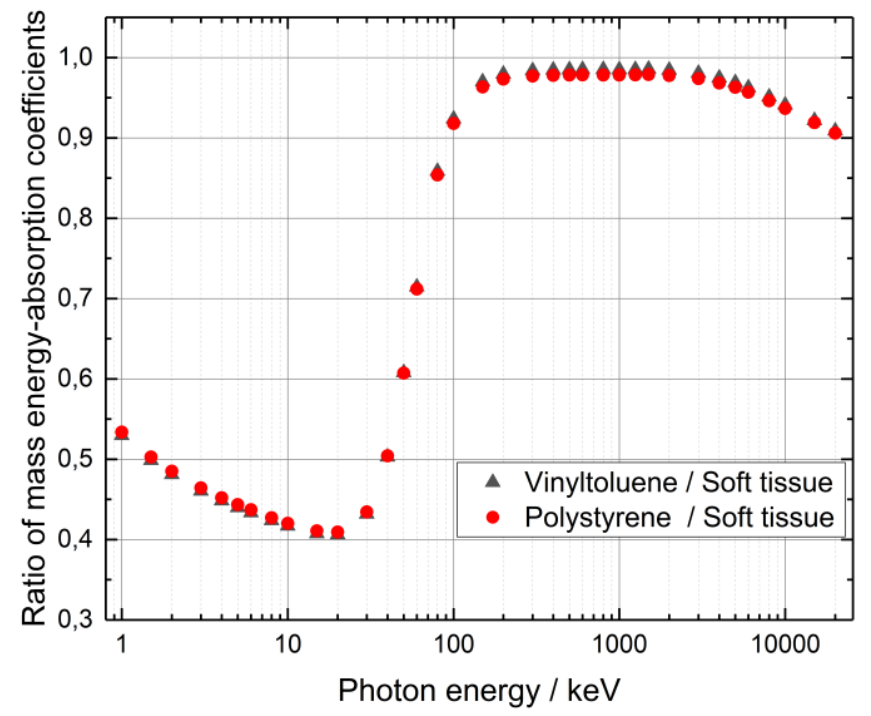

Fig. 1. Comparison of mass energy-absorption coefficients of the main polymer bases of plastic scintillators with the same for ICRU 4-component soft tissue [10].

It should be stressed that the comparison above only refers to the materials' response to the dose and does not consider the relationship between the dose measured with the detector as the whole and ambient dose equivalents which are defined in a point. Additional investigation is needed to find out how to correspond the dose measured with the detector to the value of ambient dose equivalent.

In this work, the detector (SCIONIX Holland B.V.) consists of the cylindrical crystal $\left(1^{\prime \prime} \times 1 "\right)$ of the plastic (polyvinyltoluene) scintillator EJ-200 (Eljien Technology) in an aluminum housing of a $0.4 \mathrm{~mm}$ thickness and a Hamamatsu R1924A photomultiplier with a built-in high voltage power supply.

\section{B. Data acquisition and signal processing}

The output signal from the detector is shaped, sampled and processed with a fully digital data acquisition board DAQ125 from Serious Dynamics. An event timestamp, an integral over a whole pulse, and a pulse shape are determined and transferred into a listmode file. Continuous integration gate when trigger is ready again in one sample after the last sample of the integration gate means completely non-paralyzable behavior with a known dead-time (the integration gate length plus one sample [11]). This can be implemented in dead-time corrections if the counting regime is of interest.

\section{Dosimetry Considerations}

In the energy range of $0.2-4 \mathrm{MeV}$, the absorbed dose in a plastic scintillator is directly proportional to the same in soft tissue. To estimate the energy deposition in a detector's material and, subsequently, the absorbed dose in soft tissue, the energy calibration of the detector was performed using the wide-angle Compton-coincidence technique [12].

The absorbed dose $D$ in soft tissue can be derived from the dose $D_{\text {plastic }}$ in a plastic scintillator as

$$
D=D_{\text {plastic }} / r
$$

where $r$ is the ratio of mass energy-absorption coefficients of a plastic scintillator to the same of soft tissue. For the detector's material (polyvinyltoluene) this ratio is $r=(0.981 \pm 0.004)$.

\section{Experiment}

Initially, the detector was tested in continuous radiation field. Dose rate measurements were carried out with a ${ }^{137} \mathrm{Cs}$ source with a well-known dose rate. The dose rate estimated using (1) was $(14.0 \pm 0.1) \mu \mathrm{Gy} \mathrm{h}^{-1}$ while the ambient dose rate equivalent at the same distance was stated to be $(15 \pm 2) \mu \mathrm{Sv} \mathrm{h}^{-1}$. The moderate difference between these two values can be explained that the detector is not calibrated to measure the $H^{*}(10)$.

The actual experiment was performed in pulsed radiation field at the $\gamma$ ELBE facility of the HZDR, Germany. The detector was placed in scattered radiation field that keeps the time structure of the initial beam but has an average photons' energy of hundreds keV. For this purpose, a PMMA phantom $\left(10 \mathrm{~cm} \times 10 \mathrm{~cm} \times 20 \mathrm{~cm}\right.$, the density of $\left.1.18 \mathrm{~g} / \mathrm{cm}^{3}\right)$ was used, the distance between the phantom and the detector was of $29 \mathrm{~cm}$. Such geometry of the experiment (see Fig. 2) ensures that the maximum energy of once scattered photons is less than $777 \mathrm{keV}$.

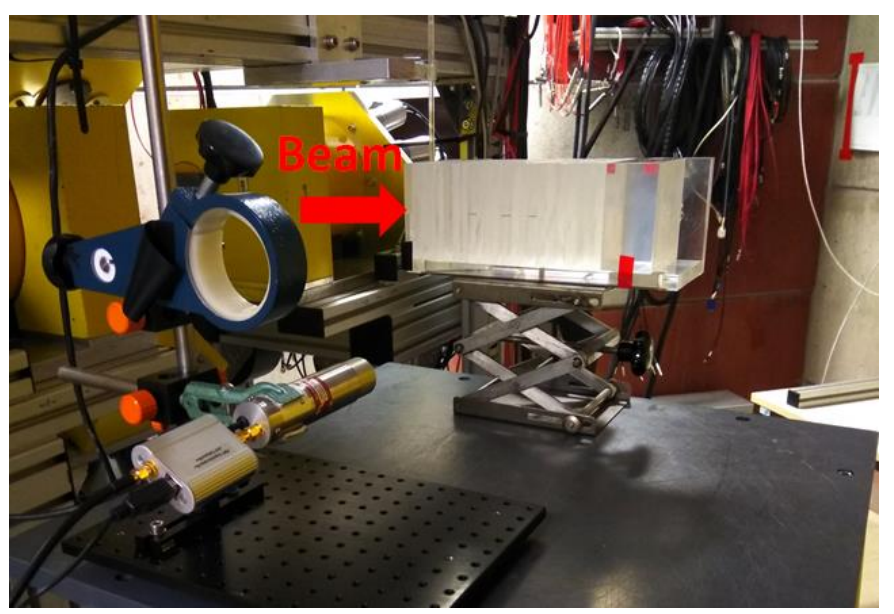

Fig. 2. Experimental setup.

In some measurements, an additional source $\left({ }^{22} \mathrm{Na}\right)$ of continuous radiation was attached to the PMMA phantom opposite to the detector. The main idea to do this was to develop and investigate an algorithm that would allow realtime distinction of pulsed and non-pulsed radiation contributions to the dose rate.

To mimic the time structure of the radiation therapy beam, 
the accelerator was working in the macropulse mode with the characteristics indicated in Table I. The DAQ125 board was synchronized to the accelerator frequency and was running with $13 \times 8$ MSamples/s.

TABLE I

\begin{tabular}{ll}
\multicolumn{1}{c}{ TIME CHARACTERISTICS OF THE BEAM } \\
\hline \hline accelerator frequency & $13 \mathrm{MHz}$ \\
micropulse period & $77 \mathrm{~ns}$ \\
macropulse duration $\Delta t$ & $5 \mu \mathrm{s}$ \\
macropulse period $T$ & $5 \mathrm{~ms}$ \\
\hline
\end{tabular}

\section{RESULTS AND DISCUSSION}

\section{A. How to Distinguish Pulsed and Non-Pulsed Radiation}

The distinguishing of pulsed and non-pulsed radiation is based on the time structure of a single interaction. Fig. 3 shows the time difference histograms for the measurement with the ${ }^{22} \mathrm{Na}$ source only, and also when the accelerator only was operated (without the ${ }^{22} \mathrm{Na}$ source attached).
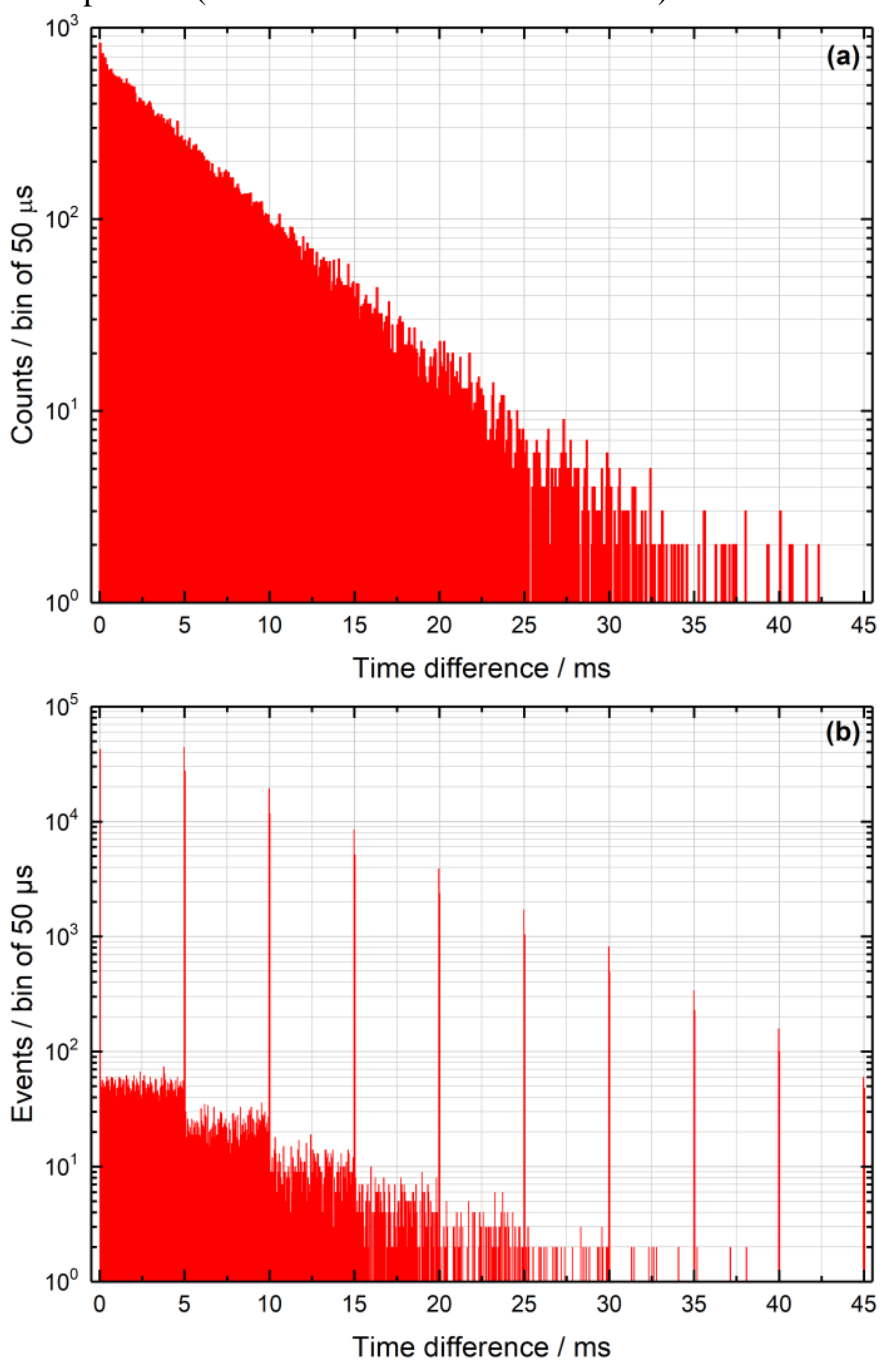

Fig. 3. Time difference histograms for the case when the ${ }^{22} \mathrm{Na}$ source only was used (a) and when the accelerator was switched on and no ${ }^{22} \mathrm{Na}$ (b). The accelerator current in (b) was of $25 \mu \mathrm{A}$.

One can see that the radiation coming from the ${ }^{22} \mathrm{Na}$ only (Fig. 3(a)) behaves according to the Poisson process, while prominent peaks with the time difference that is a multiple of the macropulse period are observed for the case when the accelerator only was working (Fig. 3(b)). One can conclude that the pulsed radiation coming from the accelerator has the time difference that belongs to the intervals of

$$
[k T-\Delta t ; k T+\Delta t]
$$

where $T$ is the macropulse period, $\Delta t$ is the macropulse duration and $k$ is used for positive integers. If one considers the events from the same macropulse, the time difference for them belongs to

$$
\text { [integration gate; } \Delta t \text { ]. }
$$

If the signal from the detected event crosses a threshold, a leading edge trigger fires, and the integration gate starts. Within the integration gate, no other trigger can occur. This means that all events within the same integration gate are not distinguished from each other and are counted as a single event which is confirmed by Fig. 4.

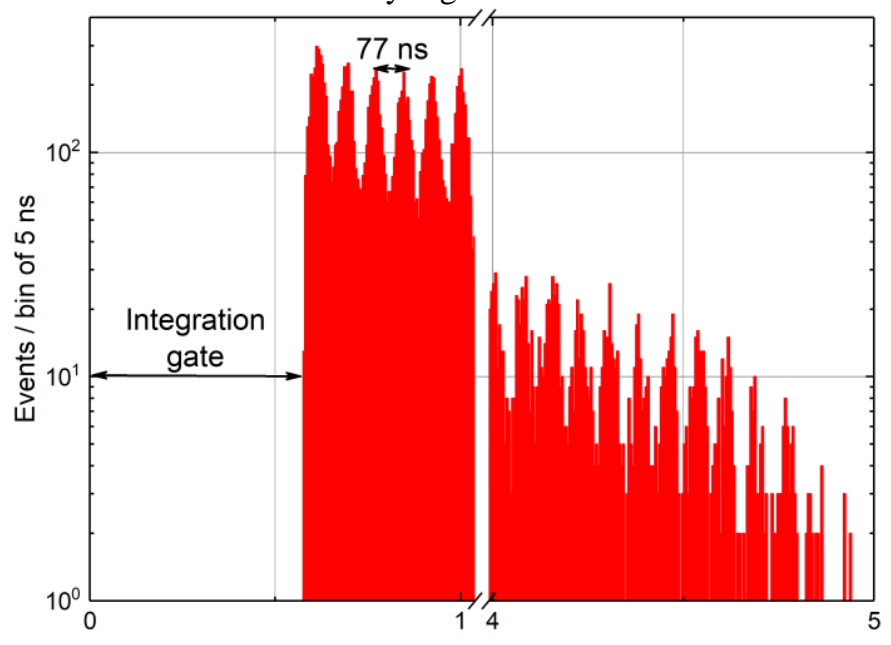

Time difference / $\mu \mathrm{s}$

Fig. 4. Enlarged first peak from Fig. 3(b) which corresponds to the time difference histogram for events from the same macropulse. No events have the time difference less that the integration gate length that was of $577 \mathrm{~ns}$ for this experiment. The small peaks are spaced by approximately $77 \mathrm{~ns}$, and this corresponds to the period of micropulses.

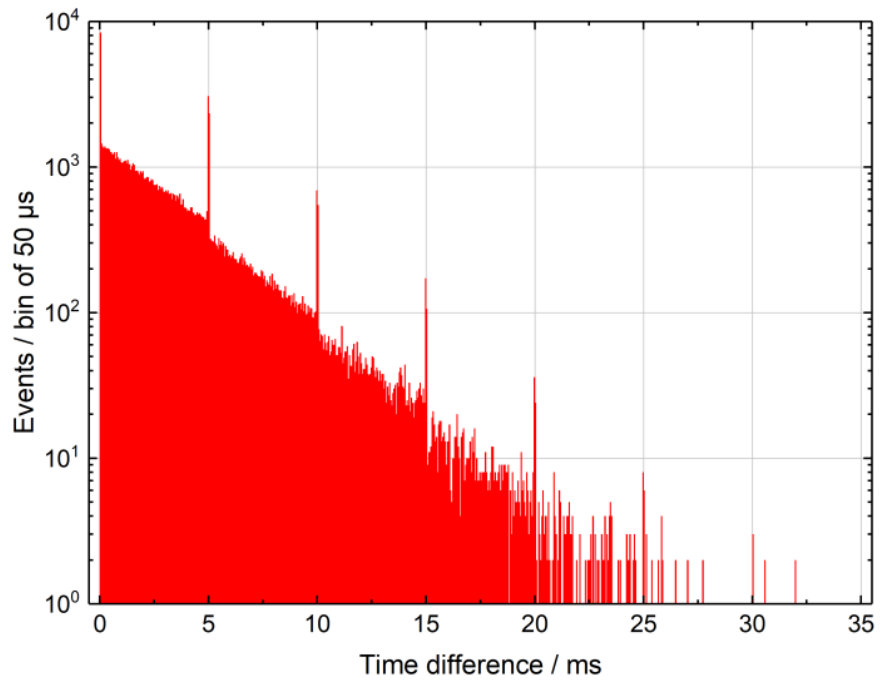

Fig. 5. Time difference histogram for the case of the accelerator switched on and with the ${ }^{22} \mathrm{Na}$ source. The accelerator current was of $25 \mu \mathrm{A}$.

Fig. 5 shows the difference histogram for case when the accelerator was operating at the current of $25 \mu \mathrm{A}$, and the ${ }^{22} \mathrm{Na}$ 
source was attached to the PMMA phantom. The interesting thing is that Fig. 5 cannot be obtained by simple summation of normalized Fig. 3(a) and Fig. 3(b). The explanation to this is that photons from the source of constant radiation can be detected almost at any time, and this leads to the decrease of the intensity of peaks with a high time difference and increases the intensity of peaks with a small time difference. In other words, the probability for the detector not to be occupied becomes lower when the source of constant radiation is used.

\section{B. Pulsed and Non-Pulsed Dose Rate Contributions to the Total Dose Rate}

The results of the total dose rate, pulsed and non-pulsed radiation dose rates are shown in Fig. 6. It is necessary to mention that Fig. 6 displays an average pulsed radiation dose rate (calculated for the whole measurement time). This means that the dose rate per pulse will be $10^{3}$ times higher.

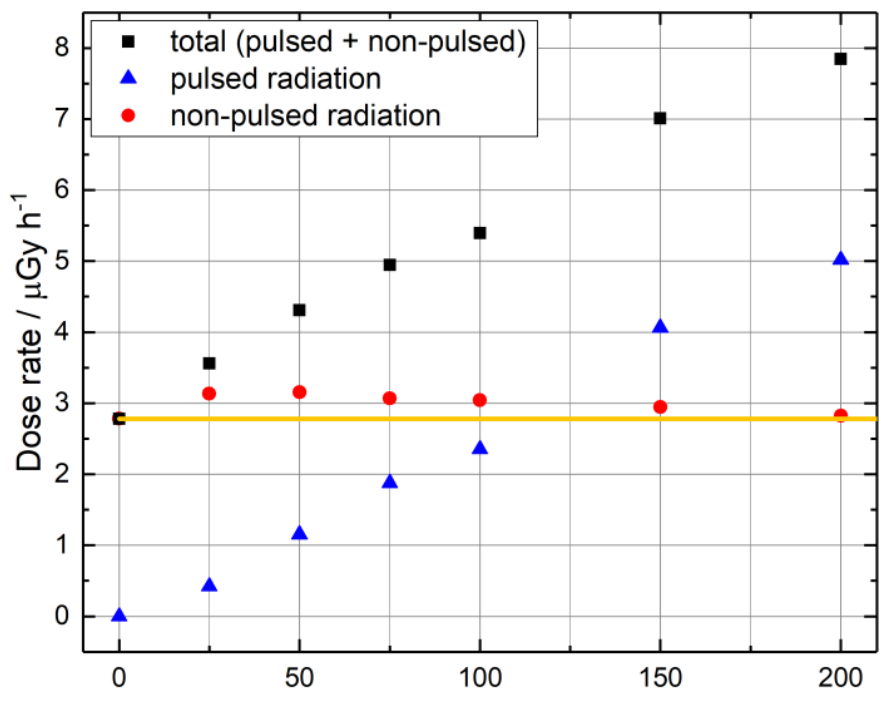

Accelerator current / $\mu \mathrm{A}$

Fig. 6. Total dose rate (assuming the ${ }^{22} \mathrm{Na}$ source and background), pulsed and non-pulsed radiation dose rates versus accelerator current. Uncertainties are enclosed within markers borders. Orange horizontal line indicates the dose rate measured for the ${ }^{22} \mathrm{Na}$ source only.

For these data, the increase of the accelerator current by $1 \mu \mathrm{A}$ leads to the increase of the total dose rate and the pulsed radiation dose rate by $(25 \pm 1) \mathrm{nGy} / \mathrm{h}$ and $(26.2 \pm 0.9) \mathrm{nGy} / \mathrm{h}$ respectively.

The behavior of the non-pulsed radiation dose rate is not constant and approaches the reference value (measured with the ${ }^{22} \mathrm{Na}$ source only) while increasing the accelerator current. This can be explained by underestimating the pulsed radiation dose rate at low accelerator current values when only one event per macropulse is detected, and this event cannot be unambiguously attributed to pulsed or non-pulsed radiation. It is supposed, that at high values of accelerator current when pulsed radiation significantly prevails over non-pulsed radiation, the influence of non-pulsed radiation contribution that belongs to the "pulsed" time intervals on the dose rate calculations can be neglected. But this question requires further investigation.

\section{CONCLUSIONS AND OUTLOOK}

The presented algorithm of distinguishing pulsed and nonpulsed radiation based on the time difference between events can be easily implemented in the software of any active dosimeter. However, there are some overestimations of pulsed radiation dose rate at low values of accelerator current. This can become significant if an additional source of non-pulsed radiation is present, and the dose rate from the source is comparable to or exceeds the pulsed radiation dose rate.

In the investigated dose rate range up to $8 \mu \mathrm{Gy} \mathrm{h}^{-1}$, the linear dependence of the total and pulsed radiation dose rate on the accelerator current is observed. But this may not work with the further increase of the accelerator current when the photons flux is so high that a time gap between two consequent integration gates leads to losses in detected events and the dose rate is underestimated. Also, the pile-up results in some losses of information about the total absorbed energy in the detector. One of the possible decisions is to expand the integration gate up to the duration of the macropulse (for example, $5 \mu \mathrm{s}$ in this work). In this case, the detector will integrate all events from the first detected within the macropulse.

One more thing to be studied is adjusting of the current detector for area monitoring when ambient dose rate equivalents $\left(\dot{H}^{*}(10)\right.$ and $\left.\dot{H}^{\prime}(0.07)\right)$ are to be measured. The corrected dose introduced in (1) cannot be treated as the operational dose, even for photon radiation the absorbed dose and the equivalent dose are numerically equal. The difference is hidden in different ways of introducing of the named doses. The corrected dose according to (1) is given for the whole detector, when the ambient dose equivalents are given in a point. A calibration to correspond the operational values is needed or even energy-dependent correction shall be required.

An additional challenge is connected with $\dot{H}^{\prime}(0.07)$ measurements. The thickness of the housing of the detector is larger than the depth in the ICRU sphere where this quantity is to be measured. This means that for measuring lowpenetrating radiation one needs housing for the detector which is equivalent to $0.07 \mathrm{~mm}$ thickness of the tissue-equivalent material or even smaller.

\section{ACKNOWLEDGMENT}

Parts of this research were carried out at ELBE at the Helmholtz-Zentrum Dresden-Rossendorf e. V., a member of the Helmholtz Association. We would like to thank the experimental facility staff for assistance.

\section{REFERENCES}

[1] Ankerhold, U., Hupe, O. and Ambrosi, P. "Deficiencies of active electronic radiation protection dosemeters in pulsed fields." Radiat. Prot. Dosim, vol. 135, no. 3, pp. 149-153, 2009, DOI:10.1093/rpd/ncp099.

[2] Gotz, M. "Dosimetry of highly pulsed radiation fields." HZDR, Dresden-Rossendorf, Germany. HZDR-90, 2018. Available: https://www.hzdr.de/publications/Publ-27317.

[3] "EPD TruDose for dosimetry in short pulsed X-ray fields using the example of industrial pulsed X-ray source XR-200." Available: https://www.thermofisher.com/order/catalog/product/EPDTRUDOSE\#/ EPDTRUDOSE. 
[4] Friedrich, S. and Hupe, O. "Dose measurements in pulsed radiation fields with commercially available measuring components." Radiat. Prot. Dosim., vol. 168, no.3, pp. 322-329, 2016, DOI:10.1093/rpd/ncv355.

[5] International Commission on Radiation Units and Measurements (ICRU) "Determination of Dose Equivalents Resulting from External Radiation Sources (Report 39)". Journal of the ICRU, vol. os20, no. 2, Feb. 1985, DOI: 10.1093/jicru/os20.2.Report39.

[6] A.R. Beierholm et al., "Investigation of linear accelerator pulse delivery using fast organic scintillator measurements." Radiat. Meas., vol. 45, no. 3-6, pp. 668-670, Mar.-Jul. 2010. DOI: 10.1016/j.radmeas.2009.11.023.

[7] Horst, F., Fehrenbacher, G. and Zink, K. "On the neutron radiation field and air activation around a medical electron LINAC." Radiat. Prot. Dosim., vol. 174, no. 2, pp. 147-158, 2017, DOI:10.1093/rpd/ncw120.

[8] Keehan, S. et al. "Dose and gamma-ray spectra from neutron-induced radioactivity in medical linear accelerators following high-energy total body irradiation." Radiat. Prot. Dosim., vol. 172, no. 4, pp. 327-332, 2016, DOI:10.1093/rpd/ncv480.

[9] Behrens, R., Pullner, B. and Reginatto, M. "X-ray emission from materials processing lasers." Radiat. Prot. Dosim., vol. 183, no. 3, pp. 361-374, 2019, DOI:10.1093/rpd/ncy126.

[10] J.H. Hubbell and S.M. Seltzer, "Tables of X-ray mass attenuation coefficients and mass energy-absorption coefficients $1 \mathrm{keV}$ to $20 \mathrm{MeV}$ for elements $Z=1$ to 92 and 48 additional substances of dosimetric interest.” NISTIR, Gaithersburg, MD, USA. Tech. Rep. NISTIR-5632, 1995.

[11] "DAQ125 Firmware (datasheet)." Serious Dynamics.

[12] K. Roemer et al., "A technique for measuring the energy resolution of low-Z scintillators.” 2009 IEEE Nucl. Sci. Symp. Conference Record (NSS/MIC), Orlando, FL, 2009, pp. 6-11, DOI: 10.1109/NSSMIC.2009.5401909. 